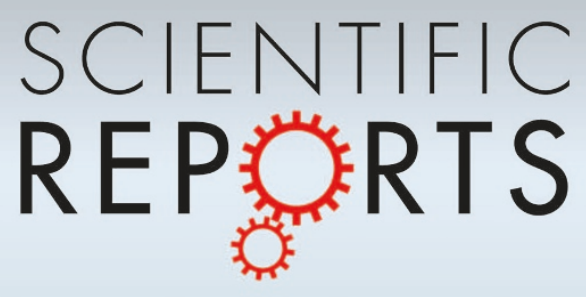

\title{
OPEN Multi-petal cyclamen flowers produced by AGAMOUS chimeric repressor expression
}

SUBJECT AREAS:

PLANT MORPHOGENESIS

PLANT MOLECULAR BIOLOGY

FLOWERING

MOLECULAR ENGINEERING IN

PLANTS

\author{
Yuri Tanaka', Yoshimi Oshima², Tomomichi Yamamura', Masao Sugiyama' , Nobutaka Mitsuda², \\ Norihiro Ohtsubo ${ }^{4}$, Masaru Ohme-Takagi ${ }^{2,3}$ \& Teruhiko Terakawa'
}

${ }^{1}$ Central Research Laboratories, Hokko Chemical Industry Co., Ltd, 2165 Toda, Atsugi, Kanagawa 243-0023, Japan,

Received

25 June 2013

Accepted

27 August 2013

Published

12 September 2013

Correspondence and requests for materials should be addressed to

T.T. (terakawa-†@ hokkochem.co.jp)

${ }^{2}$ Bioproduction Research Institute, National Institute of Advanced Industrial Science and Technology (AIST), Central 4, Higashi 1-1-1, Tsukuba, Ibaraki 305-8562, Japan, ${ }^{3}$ Institute for Environmental Science and Technology (IEST), Saitama University, Saitama 338 8770, Japan, ${ }^{4}$ NARO Institute of Floricultural Science (NIFS), National Agriculture and Food Research Organization (NARO), 2-1 Fujimoto, Tsukuba, Ibaraki 305-8519, Japan.

Cyclamen persicum (cyclamen) is a commercially valuable, winter-blooming perennial plant. We cloned two cyclamen orthologues of AGAMOUS (AG), CPAG1 and CPAG2, which are mainly expressed in the stamen and carpel, respectively. Cyclamen flowers have 5 petals, but expression of a chimeric repressor of CpAG1 (CpAG1-SRDX) caused stamens to convert into petals, resulting in a flower with 10 petals. By contrast, CpAG2-SRDX only caused incomplete formation of stamens and carpels. Expression in Arabidopsis thaliana showed similar effects on flower organ specification. Simultaneous expression of $C p A G 1-S R D X$ and $C P A G 2-S R D X$ in cyclamen induced rose-like, multi-petal flowers, a potentially valuable trait in commercial ornamental varieties. Expression of $C p A G 2-S R D X$ in a cyclamen mutant lacking expression of $C P A G 1$ more effectively produced multi-petal flowers. Here, we controlled the number of petals in cyclamen by simple genetic engineering with a chimeric repressor. This strategy may be applicable useful for other ornamental plants with two distinct $A G$ orthologues.

\footnotetext{
- lower shape and color can enhance the value of ornamental flowers. Therefore, the improvement or creation - of novel flower traits can provide substantial commercial value. The organogenesis of flowers has been well studied, especially in Arabidopsis thaliana and Antirrhinum majus. Extensive studies in these plants have revealed a common molecular mechanism of floral organ formation in seed plants, the "ABC model" model, genes encoding MADS-box transcription factors combinatorially specify flower cell fate. Class A genes, such as APETALA1 in Arabidopsis, specify the outer-most floral organs, the sepals. Class B genes such as APETALA3 and PISTILLATA specify petals in concert with class A genes. Class B genes specify male organs in concert with class $\mathrm{C}$ genes such as AGAMOUS $(A G)$. Class $\mathrm{C}$ genes specify the inner-most floral organs, the carpels. Mutation of Arabidopsis ABC genes produces homeotic transformations of one organ type into another; some of these transformations would increase plant value if they could be recapitulated in commercial ornamental varieties. For example, the flowers of Arabidopsis ag mutants have no stamen or carpel and lose the ability to terminate meristematic activity. As a result, ag mutants have "multi-petal flowers", which have a repeated structure of sepal-petal-petal including tens of petals ${ }^{4}$. Recapitulating the phenotype of ag mutants in commercial ornamental plants would be highly valuable, because this would confer both an interesting appearance and complete sterility, which would prevent the dispersal of transgenic seeds and pollen. Moreover, this trait could be produced in a selected variety with other valuable traits such as perfume or a desirable color.

Cyclamen is a winter-blooming perennial plant and one of the most popular potted flowers in many countries. The genus Cyclamen consists of about 15 species, which are distributed in the Mediterranean region. Wild-type cyclamens have five sepals, five petals, five stamens and one pistil with five fused carpels. In traditional breeding, genetic mutation has provided the only means to create multi-petal cyclamen varieties. A homeotic mutant bearing flowers with double (ten) petals and no stamen was discovered ${ }^{5}$ and commercialized, but such mutants occur at a low frequency. More interesting mutant cyclamen flowers, such as those that resemble the Arabidopsis ag mutants, with tens of petals due to failure of termination of floral meristem, are rarely observed.

We previously isolated 10 putative MADS-box transcription factor genes from cyclamen and analyzed their expression patterns ${ }^{6}$. We found that these MADS-box genes have homologs in model flowering plants such as
} 
Arabidopsis, but have distinct tissue specificities in cyclamen. Among these genes, we identified CpAG1 and CpAG2 (AB548889 and AB548890), two closely related class C paralogs similar to Arabidopsis AG. CpAG1 and CpAG2 share 90\% amino acid identity. In Arabidopsis, transcripts of $A G$ are expressed in whorls 3 and $4^{4,7,8}$. In cyclamen, transcripts of CPAG1 were mainly detected in the stamen, and CPAG2 expression was mainly detected in the carpel, although the transcripts of each gene were also detected in stamen, carpel and receptacle ${ }^{6}$.

Chimeric repressor gene-silencing technology (CRES-T) provides a powerful tool for dominant negative genetic modification of a transcription factor. In CRES-T, a short but strong, plant-specific transcriptional repression domain (SRDX) is fused to the coding sequence of transcription factor of interest and expressed in plants 9 . The resulting chimeric repressor can suppress the expression of the transcription factor's target genes and thus induce a dominant negative phenotype of the transcription factor, even in the presence of other functionally redundant transcription factors. For example, introduction of a chimeric repressor of $A G$ or its ortholog efficiently induced an ag-like multi-petal flower phenotype in Arabidopsis and Pharbitis nil (morning glory) $)^{10,11}$. However, the manipulation of $P$. nil required use of the $P$. nil $A G$ ortholog and expression from an inducible promoter to avoid developmental lethality ${ }^{11}$.

In this study, we succeeded in controlling the number of petals (5, 10 and $>40$ ) of cyclamen by using CRES-T constructs for CPAG1 and $C p A G 2$. Furthermore, we showed that the two closely related cyclamen class $C$ genes $C p A G 1$ and $C p A G 2$ have divergent functions in cyclamen flower development; $C p A G 1$ mainly functions to produce stamen and $C P A G 2$ functions in whorl 4 to produce carpels and terminate the floral meristem. Our findings improve our understanding of flower formation in cyclamen and provide an efficient strategy to control petal number in ornamental plants that show similar subfunctionalization of $A G$ orthologues.

\section{Results}

Expression profiles of cyclamen class $C A G$ homologs. To obtain insights into the functions of $C p A G s$, as part of our strategy to produce multi-petal cyclamen flowers, we analyzed the expression patterns of CPAG1 and CPAG2 in wild type floral organs (Fig. 1a). We found that CPAG1 is predominantly expressed in stamen in addition to low expression in carpel, and CpAG2 is expressed mainly in carpel but rarely detectable in stamen (Fig. 1b). These data suggest that $C p A G 1$ and $C p A G 2$ mainly function in stamen and carpel, respectively.

To evaluate the involvement of CPAG1 and CPAG2 in the formation of floral organs, we analyzed their expression by RT-PCR in wild type and mutant varieties. For example, we examined the WP strain, which contains a mutation causing a homeotic transformation resulting in flowers with 10 petals. We detected expression of both genes in wild type, but only CPAG2 was detected in WP and other similar mutant strains with 10 petals (Fig. 1c). This suggests that $C p A G 1$ is involved in stamen formation and the lack of CPAG1 expression causes the homeotic conversion of stamen into petal in WP and other 10- petals varieties.

Expression of CpAG1 and CpAG2 chimeric repressors in Arabidopsis. To examine the functions of cyclamen class $\mathrm{C}$ genes and determine rapidly which construct is most suitable to induce the desired morphological changes, we first tested CpAG function in transgenic Arabidopsis. To create dominant negative mutants, we expressed CpAG1 and CpAG2 chimeric repressors under the control of the CaMV 35S promoter (35S:CPAG1/2-SRDX). The 35S:CpAG1-SRDX Arabidopsis plants showed petaloid stamens but had a less prominent effect on carpels, suggesting that the class $C$ function of $A G$ in stamen was impaired (Fig. 2b, $\mathrm{f}$ to $\mathrm{h}$ ). By contrast, 35S:CpAG2-SRDX Arabidopsis plants had abnormal carpels and

\section{a}
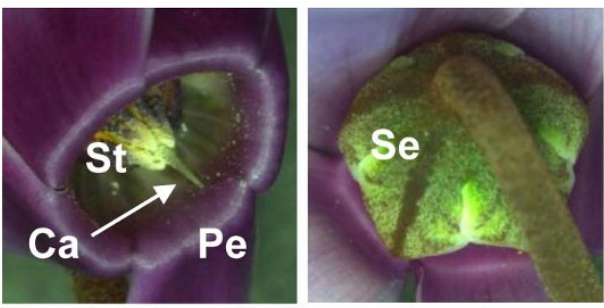

$b$
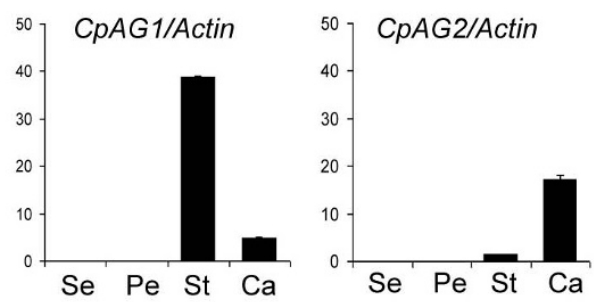

C

\begin{tabular}{|c|c|c|c|c|c|c|c|}
\hline \multicolumn{4}{|c|}{ Wild type } & \multicolumn{4}{|c|}{ Double petal } \\
\hline MW & MP & MB & SP & WP & WW & WB & WPu \\
\hline
\end{tabular}

CpAG1

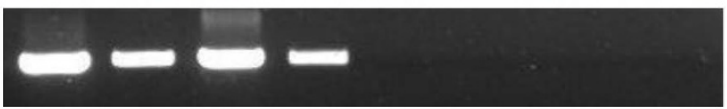

CpAG2

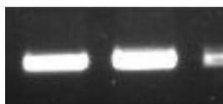

CpActin

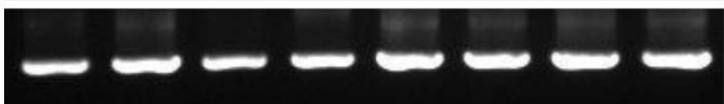

Figure 1 | Expression of $C p A G 1$ and $C p A G 2$. (a) Floral organs of wildtype cyclamen. Se, sepal; Pe, petal; St, stamen; Ca, carpel. (b) Quantitative analysis of $C p A G 1$ and $C p A G 2$ expression in floral organs of wild type by RT-qPCR. (c) Expression of $C p A G 1$ and $C p A G 2$ in various strains, with five petals; MW: Mini White; MP: Mini Pink; MB: Mini Blue; and SP: Salmon Pink, and with 10 petals; WP: Wink Pink; WW: Wink White; WB: Wink Blue; and WPu: Wink Purple. All photographs were taken by the authors and the figure copyright is reserved by the authors.

ectopic formation of carpels and stamens in addition to abnormal stamens, but at a lower rate than 35S:CpAG1-SRDX (Fig. 2c, $\mathrm{f}$ to $\mathrm{h}$ ). This phenotype in carpels indicates that another important role of $A G$, termination of meristematic activity, was perturbed. The Arabidopsis AG chimeric repressor also induced these phenotypes in mild-phenotype lines of Arabidopsis in addition to inducing a complete ag-like phenotype in stronger lines ${ }^{10,12}$. Thus, $C p A G 1$ and $C p A G 2$ appeared to have at least some of the same roles as $A G$ in Arabidopsis.

Arabidopsis has only one class $\mathrm{C}$ gene in its genome, but cyclamen has at least two class $C$ genes, $C p A G 1$ and $C p A G 2$. To examine whether these two $C p A G s$ have a synergetic effect, we introduced 35S:CpAG1-SRDX and 35S:CpAG2-SRDX together into Arabidopsis. A significant portion of 35S:CpAG1-SRDX 35S:CpAG2-SRDX Arabidopsis plants showed a multi-petal phenotype similar to the ag mutants (Fig. 2d to h), suggesting that CpAG1 and CpAG2 might have slightly different functions. Specifically, $C p A G 1$ acts mainly in stamen formation and $C p A G 2$ acts mainly in carpel formation and termination of mersitematic activity, but together they add up to complete $A G$ function.

Morphologies of 35S:CPAG1-SRDX and 35S:CpAG2-SRDX cyclamen. To produce transgenic cyclamen with multi-petal flowers, we first introduced 35S:AG-SRDX into cyclamen. However, unlike 

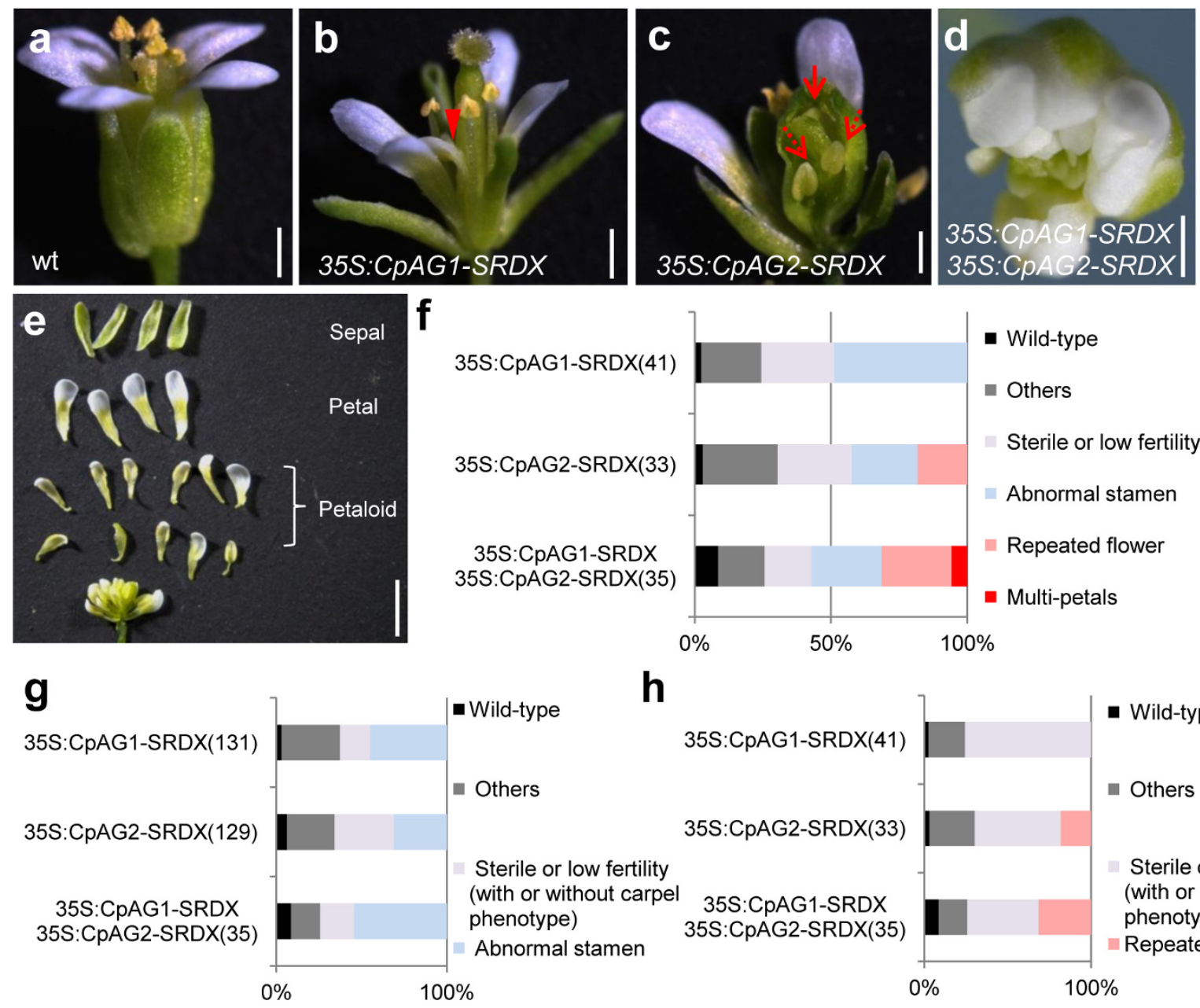

h

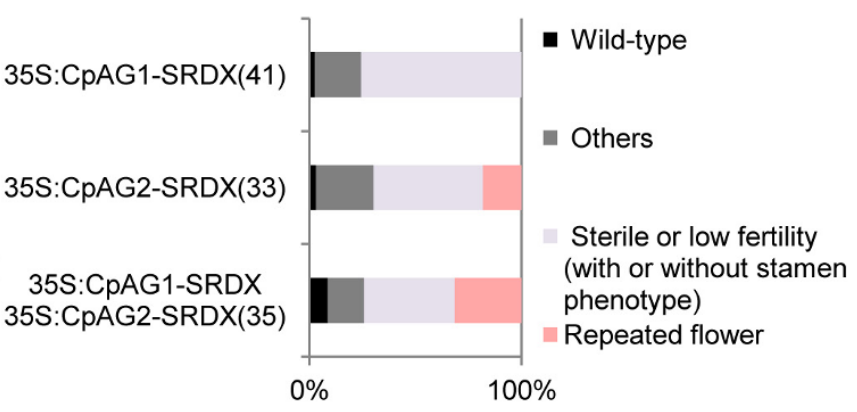

Figure 2 Phenotypes of 35S:CpAG1-SRDX and 35S:CpAG2-SRDX Arabidopsis. (a) A flower of wild-type Arabidopsis. (b) A flower of 35S:CpAG1SRDX Arabidopsis with petaloid stamens (red arrowhead). (c) A double flower of 35S:CPAG2-SRDX plant. Inside the carpel is shown by dissection. Solid arrow and dashed arrows indicate ectopically formed carpel and stamen, respectively, in an original carpel. (d) A multi-petal flower of 35S:CPAG1-SRDX 35S:CPAG2-SRDX plant. (e) The multi-petal flower of (d) has repeated petaloids. (f) The frequency of each phenotype class in 35S:CPAG1-SRDX, 35S:CPAG2-SRDX and 35S:CPAG1-SRDX 35S:CPAG2-SRDX plants. "Abnormal stamen" includes immature-like stamen and petaloid stamen. The abnormal stamen phenotype with carpel alteration is included in "Repeated flower". "Multi-petals" includes triple or more repeated flowers with petaloid stamens. (g) The frequency of stamen alteration in 35S:CpAG1-SRDX, 35S:CpAG2-SRDX and 35S:CpAG1-SRDX 35S:CpAG2-SRDX plants. The frequencies of abnormal stamen were significantly different between 35S:CpAG1-SRDX and 35S:CpAG2-SRDX (p < 0.05, Fisher's exact test). (h) The frequency of carpel alteration (repeated flower) in 35S:CpAG1-SRDX, 35S:CpAG2-SRDX and 35S:CpAG1-SRDX 35S:CpAG2-SRDX plants. The number of examined plants is given in parentheses in each case. Bars indicate $0.5 \mathrm{~mm}$ in (a) to (d) and $2 \mathrm{~mm}$ in (e). All photographs were taken by the authors and the figure copyright is reserved by the authors.

35S:AG-SRDX Arabidopsis, 35S:AG-SRDX did not alter floral morphology in 7 transgenic cyclamen lines that showed clear expression of the transgene (Fig. S1). This result indicated that Arabidopsis AG cannot substitute for $C p A G 1$ and $C p A G 2$ in cyclamen in our experiments. This is consistent with reports that 35S:AG-SRDX did not induce the expected phenotypes in other ornamental plants ${ }^{13,14}$.

Next, we introduced 35S:CpAG1-SRDX and 35S:CpAG2-SRDX into MW and MR wild-type cyclamen, which have 5 petals in their flowers. Two out of 8 transgenic cyclamen lines expressing 35S:CpAG1-SRDX produced double-petal (10-petal) flowers, with an additional 5 petals (Fig. 3a, b, d) instead of stamens. Other features such as number of carpel and sepals, petal size, leaf shape were normal (Fig. 3a, b). This phenotype of two independent transgenic lines is similar to that of the cyclamen WP strain in which the expression of CPAG1 is suppressed. These data suggest that CRES$\mathrm{T}$ efficiently perturbed CpAG1 function and this strategy can produce double-petal flowers in cyclamen. By contrast, 4 out of 11 transgenic cyclamen lines expressing 35S:CpAG2-SRDX showed abnormal or petaloid stamens (Fig. 3c, d). However, these lines exhibited neither complete conversion of stamen into petal nor indeterminate growth of the floral meristem (Fig. 3c, d).

To obtain multi-petal flowers, as was observed in Arabidopsis, we suppressed both CpAG1 and CpAG2 by two strategies. First, we introduced 35S:CpAG2-SRDX into WP, which lacks CPAG1 expression. We found that 28 out of $3935 S$ :CPAG2-SRDX WP plants produced multi-petal flowers with a repeated structure of tens of petals ( $>40$ petals) instead of stamens and carpel (Fig. 3e, g-i). Second, we produced transgenic cyclamen lines that harbor 35S:CPAG1-SRDX and 35S:CpAG2-SRDX in the MB wild-type background, which has 5 normal petals. We found 3 out of 22 35S:CpAG1-SRDX 35S:CpAG2$S R D X$ cyclamen also produced multi-petal flowers similar to $35 S$ : CPAG2-SRDX WP plants (Fig. 3f). Thus, we can produce multi-petal flowers by expressing two chimeric AG repressors in wild-type cyclamen. 

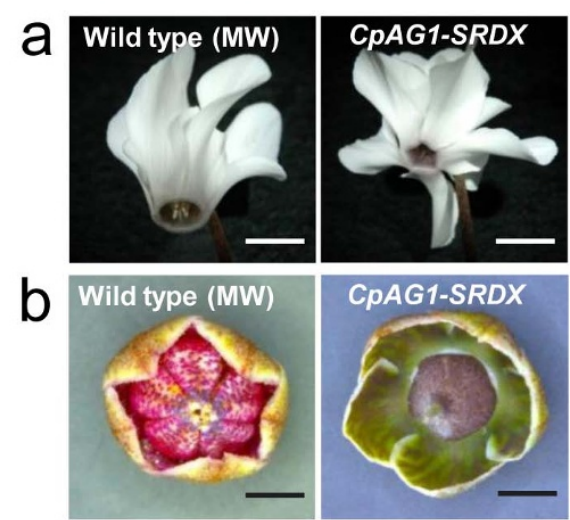

C

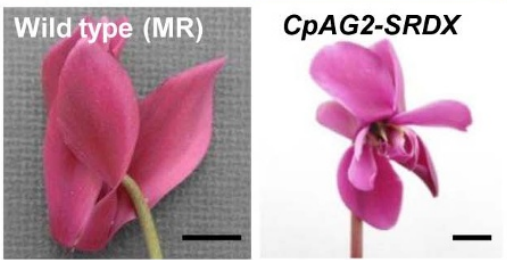

d

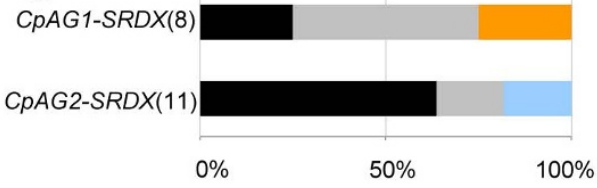

wild-type Others

Petaloid stamen Double petal
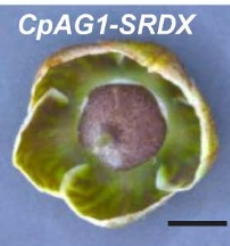

Double-petal (WP)

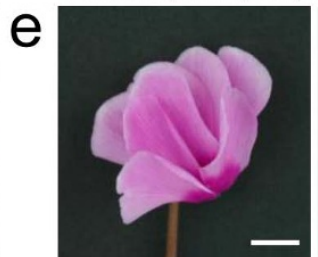

f
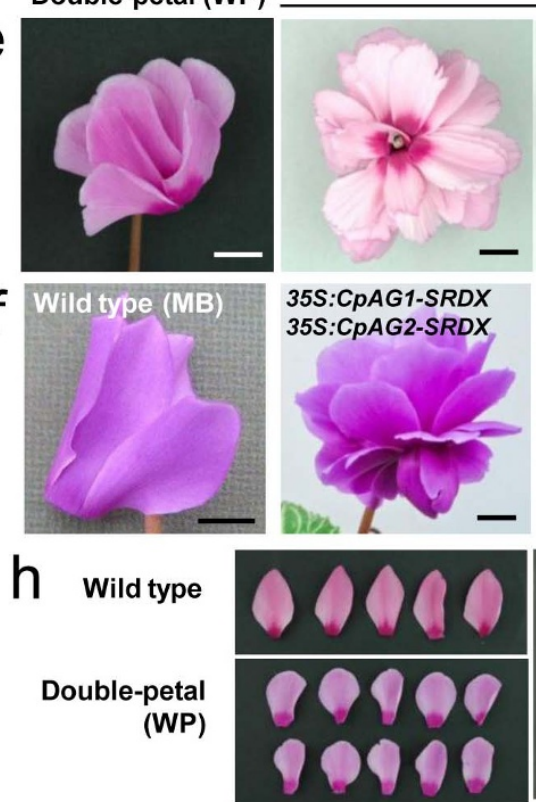

CpAG2-SRDX WP
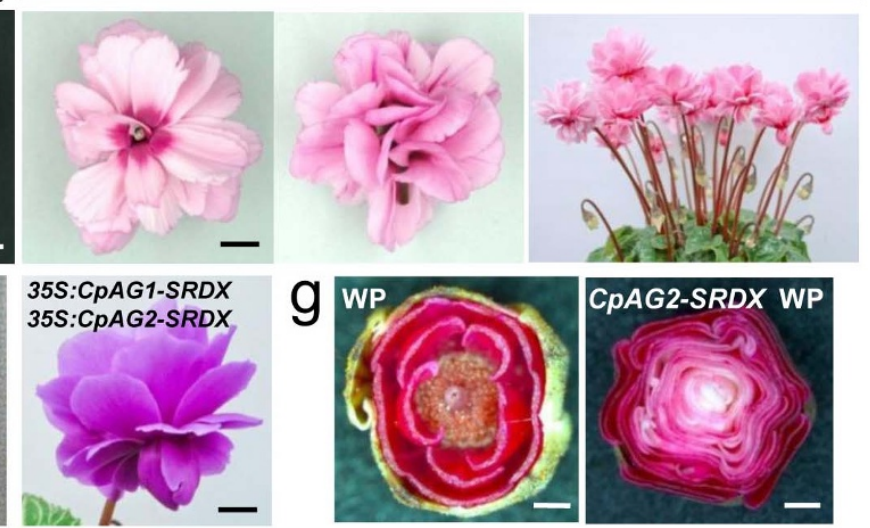

i 35S:CPAG1-SRDX 35S:CpAG2-SRDX(39) 35S:CPAG2-SRDX WP (22)

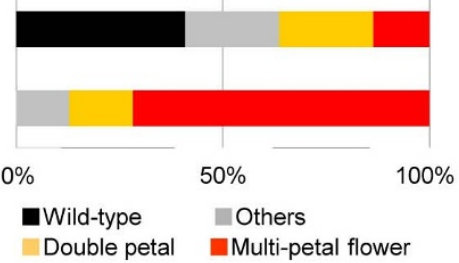

Figure 3 | Phenotypes of 35S:CPAG1-SRDX and 35S:CPAG2-SRDX cyclamen. (a) A flower of a MW wild-type plant (left) and a double-petal flower of a 35S:CpAG1-SRDX plant (right). (b) A flower of MW wild-type (left) and 35S:CpAG1-SRDX (right) plants, with the petals removed. The 35S:CpAG1SRDX plant has no stamen. (c) A flower of an MR wild type plant (left) and a flower of a 35S:CpAG2-SRDX plant with abnormal petaloid stamens (right). (d) The frequency of each phenotype in 35S:CPAG1-SRDX and 35S:CPAG2-SRDX plants. The number of examined plants is given in parentheses in each case. (e) A double-petal flower of WP and a multi-petal flower of a 35S:CpAG2-SRDXWP plant. The view from top (left), bottom (middle), and the whole plant (right). (f) A flower of a 35S:CPAG1-SRDX 35S:CpAG2-SRDX plant (MB background). (g) Horizontal sections of flowers of WP (left) and 35S:CPAG2-SRDXWP (right). The 35S:CPAG2-SRDXWP plant has neither stamen nor carpel and produced tens of petals. (h) The petals of wild type, a double-petal mutant and 35S:CPAG2-SRDX WP plants. (i) The frequency of each phenotype in 35S:CpAG1-SRDX 35S:CpAG2-SRDX (MB background) and 35S:CPAG2-SRDXWP plants. Bars indicate $10 \mathrm{~mm}$ in (a), (c), (e), (f) and $2 \mathrm{~mm}$ in (b), (g). All photographs were taken by the authors and the figure copyright is reserved by the authors.

\section{Discussion}

Here we describe the production of new transgenic cyclamen varieties with double-petal (10-petals) and multi-petal ( $>40$ petals) flowers by perturbing the functions of CpAG1 and CpAG2 with CRES-T. Even though the amino acid sequences of CpAG1 and CpAG2 are quite similar, the effects of CpAG1-SRDX and CpAG2-SRDX are quite different and synergistic. This may be due to the following reasons: First, our evidence indicates that CpAG1 and CpAG2 have slightly different functions. For example, constitutive expression of CPAG1-SRDX induced petals instead of stamens, but expression of $C P A G 2-S R D X$ did not induce an increase in petals in cyclamen. $C P A G 2-S R D X$ was also less effective than CPAG1-SRDX in inducing abnormal stamens in Arabidopsis. Therefore, we conclude that CpAG1 and CpAG2 have similar protein functions but have their own distinct roles in whorl 3 and whorl 4, respectively. The examination of how such paralogous transcription factors exert their own distinct functions, probably with slight different binding-site sequence specificities, will provide an interesting topic for future research.

In addition to differences in protein function, differences in gene expression may also affect $C p A G 1$ and $C p A G 2$ functions. For example $C p A G 1$ and $C p A G 2$ were detected in carpels, but $C p A G 1$ is mainly expressed in stamens. Thus, we hypothesized that $C P A G 1$ regulates stamen (whorl 3) development and CpAG1 and CpAG2 redundantly regulate the whorl 4 development because: First, the expression of CPAG1 is preferably detected in stamens and not detected in WP, which has 10 petals and no stamen. Second, no mutant showing defects only in whorl 4 has been reported to date in cyclamen. Third, introduction of CPAG2-SRDX into WP, which lacks expression of $C P A G 1$, effectively induced an increased number of petals instead of carpels and indeterminate growth of the floral meristem; this was more effective than the simultaneous or sole introduction of CPAG1-SRDX and CPAG2-SRDX into wild type. We consider that the genetic redundancy of CPAG1 and CPAG2 in whorl 4 was not fully overcome by the introduction of chimeric repressor(s) into wild type. By contrast, the introduction of CpAG1$S R D X$ into wild-type was effective enough to induce the same phenotype observed in WP, probably because of a lack of genetic redundancy in the stamen.

During the evolution of flowering plants, duplication of MADSbox genes was followed by gene loss, neofunctionalization and subfunctionalization by changes in transcriptional regulation and protein sequence in different lineages ${ }^{15,16}$. In core eudicots, $C$ lineage MADS-box genes have separated into euAG and PLENA (PLE) lineages ${ }^{17}$. After duplication, the primary $C$ functions were 
subfunctionalized $^{15}$. For example, in Antirrhinum majus, PLE has a main role in $\mathrm{C}$ functions, including termination of floral meristem and establishment of stamen and carpel identities, but the euAG sublineage gene FARINERRI (FAR) contributes only to male fertility. The differences in these genes reside not only in their expression patterns but also in their protein functions ${ }^{18}$. In Nicotiana benthamiana, euAG- and PLE-like genes redundantly contribute to all class-C functions ${ }^{19}$. The class- $C$ function in monocots is also divided between two genes, but in a distinct manner. Rice OsMADS 3 and OsMADS58 play a more predominant role in stamen and carpel identity and meristem termination, respectively ${ }^{20}$. This subfunctionalization appears to rely on differences in protein function such as specificity of protein-protein interactions, but not on differences in expression patterns ${ }^{20}$. The situation in cyclamen is somewhat similar to that of rice where the genes that mainly function in stamen and carpel are different. However, this subfunctionalization of CPAG1 and $C p A G 2$, both of which belong to the euAG sub-lineage, occurred very recently in evolution, because no other similar subfunctionalization has been reported in dicots.

In this study, we successfully produced double- and multi-petal cyclamen flowers by regulating the function of CPAGs with CRES-T. The chimeric repressor constructs may save time in breeding novel double- and multi-petal cyclamen varieties based on preexisting cultivars with valuable characteristics such as perfume, ruffled petals and attractive colors. Our strategy to create multi-petal flowers may be able to be implemented in other ornamental plants with similar class $\mathrm{C}$ subfunctionalization.

\section{Methods}

Plant materials. Cyclamen persicum variety Mini White (MW), Mini Red (MR), Mini Purple (MP) and Mini Blue (MB) were used for wild-type control plants. The varieties of Wink Pink (WP) and Wink White (WW) were homeotic mutants which have flowers with 10 petals. For the experiments in Arabidopsis, Arabidopsis thaliana ecotype Columbia-0 (Col-0) was grown in Petri dishes or on soil under 16/8 hr light/ dark cycle at $23^{\circ} \mathrm{C}$.

Expression analysis of $C p A G 1$ and $C p A G 2$. Floral buds of wild-type cyclamen and homeotic mutant were collected and used for RNA extraction and semi-quantitative RT-PCR (RTsqPCR). Total RNA was extracted and treated with DNase as described previously ${ }^{6}$. These RNA samples were subjected to RTsqPCR using PrimeScript One Step RT-PCR Kit Ver.2 (Takara Bio Inc., Japan).

For quantitative analysis, total RNAs were isolated from sepals, petals, stamens and carpels of wild-type flowers and were treated with DNase as described above. These RNA samples were used for reverse transcription by using High Capacity cDNA Reverse Transcription Kit (Life Technologies Inc., USA) according to the manufacturer's instructions. Quantitative real-time RT-PCR with three biological replicates was carried out using Applied Biosystems 7300 real-time PCR system and Power SYBR Green PCR Master Mix (Life Technologies Inc., USA). Since the coding sequences of $C p A G 1$ and $C p A G 2$ gene are very similar, gene-specific primers were designed in their $3^{\prime}$ UTRs.

Plasmid construction. The full-length coding regions of CPAG1 and CPAG2 were cloned from C. persicum cvs. MP and used in this study. The 35S:CpAG1-SRDX and 35S:CpAG2-SRDX constructs were prepared as described previously ${ }^{10}$. To prepare the construct which has both 35S:CpAG1-SRDX and 35S:CpAG2-SRDX, the 35S:CpAG1$S R D X$ fragment was amplified by PCR using primers with attB4 and attB1R sequence (\#4155; 5' -ggggacaactttgtatagaaaagttgaaGGCGCCGGAACCAATTAAGCTT-3', \#4156; 5' -ggggactgcttttttgtacaaacttggCGATCTAGTAACATAGATGACAC-3') and was cloned into pDONRG-P4P1R 12 by Gateway BP reaction (Life Technologies, USA). The resultant entry clone and 35S:CPAG2-SRDX cloned in regular entry clone were assembled in R4pGWB501 ${ }^{21}$ by multi-site Gateway LR reaction (Life Technologies).

Transformation of cyclamen and Arabidopsis. The binary vector plasmids 35S:CpAG1-SRDX and/or 35S:CpAG2-SRDX pBCKH were introduced into Agrobacterium tumefaciens strain GV3101 and LBA4404. Agrobacterium-mediated transformations of Arabidopsis and cyclamen were performed as described ${ }^{22,23}$. To confirm genetic transformation, total DNA was extracted from the leaf tissue of putative shoots using cetyltrimethylammonium bromide $(\mathrm{CTAB})^{24}$. Approximately $10 \mathrm{mg}$ of leaf tissues were homogenized with $200 \mu \mathrm{l}$ of CTAB buffer. Integration of HPT was confirmed by PCR with the specific primers: 5'-ATGAAAAAGCCTGAACTCACCGCGA-3' and 5'-TCCATCACAGTTTGCCAGTGATACA-3'. The plantlets were transplanted into pots containing growing soil with vermiculite and perlite, and were grown in a growth chamber at $20^{\circ} \mathrm{C}$ with $16 \mathrm{~h}$ of light per day. For flowering, transgenic plants were grown in a closed, special-netted greenhouse.
1. Coen, E. S. \& Meyerowitz, E. M. The war of the whorls: Genetic interactions controlling flower development. Nature 353, 31-37 (1991).

2. Irish, V. F. The flowering of Arabidopsis flower development. Plant J. 61, 1014-1028 (2010).

3. Krizek, B. A. \& Fletcher, J. C. Molecular mechanisms of flower development: an armchair guide. Nat. Rev. Genet. 6, 688-698 (2005).

4. Yanofsky, M. F. et al. The protein encoded by the Arabidopsis homeotic gene agamous resembles transcription factors. Nature 346, 35-39 (1990).

5. Grey-Wilson, C. Aberrations. Cyclamen: A Guide for Gardeners, Horticulturists and Botanists. 198-199 (B T Batsford, London, 2002).

6. Tanaka, Y., Yamamura, T. \& Terakawa, T. Identification and expression analysis of the Cyclamen persicum MADS-box gene family. Plant Biotechnol 28, 167-172 (2011).

7. Davies, B. et al. PLENA and FARINELLI: redundancy and regulatory interactions between two Antirrhinum MADS-box factors controlling flower development. EMBO J. 18, 4023-4034 (1999).

8. Riechmann, J. L. \& Meyerowitz, E. M. MADS domain proteins in plant development. Biol. Chem. 378, 1079-1101 (1997).

9. Hiratsu, K., Matsui, K., Koyama, T. \& Ohme-Takagi, M. Dominant repression of target genes by chimeric repressors that include the EAR motif, a repression domain, in Arabidopsis. Plant J. 34, 733-739 (2003).

10. Mitsuda, N. et al. Efficient production of male and female sterile plants by expression of a chimeric repressor in Arabidopsis and rice. Plant Biotechnol. J. 4, 325-332 (2006)

11. Sage-Ono, K. et al. Induction of double flowers in Pharbitis nil using a class-C MADS-box transcription factor with Chimeric REpressor gene-Silencing Technology. Plant Biotechnol. 28, 153-165 (2011).

12. Oshima, Y. et al. Novel vector systems to accelerate functional analysis of transcription factors using chimeric repressor gene-silencing technology (CRES-T). Plant Biotechnol. 28, 201-210 (2011).

13. Narumi, T. et al. Chimeric AGAMOUS repressor induces serrated petal phenotype in Torenia fournieri similar to that induced by cytokinin application. Plant Biotechnol. 25, 45-53 (2008).

14. Mitsuda, N. et al. The new fioreDB database provides comprehensive information on plant transcription. Plant Biotechnol. 28, 123-130 (2011).

15. Causier, B. et al. Evolution in action: following function in duplicated floral homeotic genes. Curr. Biol. 15, 1508-1512 (2005).

16. Airoldi, C. A. \& Davies, B. Gene duplication and the evolution of plant MADS-box transcription factors. J. Genet. Genomics 39, 157-165 (2012).

17. Kramer, E. M., Jaramillo, M. A. \& Di Stilio, V. S. Patterns of gene duplication and functional evolution during the diversification of the AGAMOUS subfamily of MADS box genes in Angiosperms. Genetics 166, 1011-1023 (2004).

18. Davies, B. et al. PLENA and FARINELLI: redundancy and regulatory interactions between two Antirrhinum MADS-box factors controlling flower development. EMBO J. 18, 4023-4034 (1999).

19. Fourquin, C. \& Ferrandiz, C. Functional analyses of AGAMOUS family members in Nicotiana benthamiana clarify the evolution of early and late roles of C-function genes in eudicots. Plant J. 71, 990-1001 (2012).

20. Yamaguchi, T. et al. Functional diversification of the two C-class MADS box genes OSMADS3 and OSMADS58 in Oryza sativa. Plant Cell 18, 15-28 (2006).

21. Nakagawa, T. et al. Development of R4 gateway binary vectors (R4pGWB) enabling high-throughput promoter swapping for plant research. Biosci. Biotechnol. Biochem. 72, 624-629 (2008).

22. Clough, S. J. \& Bent, A. F. Floral dip: a simplified method for Agrobacterium mediated transformation of Arabidopsis thaliana. Plant J. 16, 735-743 (1998).

23. Tanaka, Y. et al. Creating ruffled flower petals in Cyclamen persicum by expression of the chimeric cyclamen TCP repressor. Plant Biotechnology 28, 141-147 (2011).

24. Murray, M. G. \& Thompson, W. F. Rapid isolation of high molecular weight plant DNA. Nucleic Acids Res. 8, 4321-4325 (1980).

\section{Acknowledgments}

This work was supported by the Program for Promotion of Basic and Applied Researches for Innovations in Bio-oriented Industry from the Bio-oriented Technology Research Advancement Institution and also supported by the Research and Development Project for Application in Promoting New Policy of Agriculture, Forestry and Fisheries.

\section{Author contributions}

Y.T. and Y.O. performed all experiments in cyclamen and Arabidopsis, respectively. T.Y. and M.S. helped experiments in cyclamen. Y.T., Y.O., N.M. and T.T. analyzed all data. Y.T., Y.O., N.M., M.O.T., N.O. and T.T. wrote the paper. M.O.T., N.O. and T.T. supervised the entire project.

\section{Additional information}

Supplementary information accompanies this paper at http://www.nature.com/ scientificreports

Competing financial interests: The authors declare no competing financial interests. 
How to cite this article: Tanaka, Y. et al. Multi-petal cyclamen flowers produced by AGAMOUS chimeric repressor expression. Sci. Rep. 3, 2641; DOI:10.1038/srep02641 (2013) cc) (i) $\odot$ This work is licensed under a Creative Commons AttributionBY No NonCommercial-NoDerivs 3.0 Unported license. To view a copy of this license, visit http://creativecommons.org/licenses/by-nc-nd/3.0 